\title{
Structural topology and shape optimization for a frequency response problem
}

\author{
Z.-D. Ma $^{1}$, N. Kikuchi' ${ }^{2}$, I. Hagiwara ${ }^{3}$ \\ 1 Department of Applied Mechanics, Jilin University of Technology, Changchun, Peoples Republic of China \\ 2 Computational Laboratory, Department of Mechanical Engineering and Applied Mechanics, The University of Michigan, \\ Ann Arbor, MI 48109-2215, USA \\ 3 Vehicle Research Laboratory, Central Engineering Laboratories, Nissan Motor Co., LTD, Japan
}

\begin{abstract}
A topology and shape optimization technique using the homogenization method was developed for stiffness of a linearly elastic structure by Bendsøe and Kikuchi (1988), Suzuki and Kikuchi (1990, 1991), and others. This method has also been extended to deal with an optimal reinforcement problem for a free vibration structure by Diaz and Kikuchi (1992). In this paper, we consider a frequency response optimization problem for both the optimal layout and the reinforcement of an elastic structure. First, the structural optimization problem is transformed to an Optimal Material Distribution problem (OMD) introducing microscale voids, and then the homogenization method is employed to determine an equivalent "averaged" structural analysis model. A new optimization algorithm, which is derived from a Sequential Approximate Optimization approach (SAO) with the dual method, is presented to solve the present optimization problem. This optimization algorithm is different from the CONLIN (Fleury 1986) and MMA (Svanderg 1987), and it is based on a simpler idea that employs a shifted Lagrangian function to make a convex approximation. The new algorithm is called "Modified Optimality Criteria method (MOC)" because it can be reduced to the traditional OC method by using a zero value for the shift parameter. Two sensitivity analysis methods, the Direct Frequency Response method (DFR) and the Modal Frequency Response method (MFR), are employed to calculate the sensitivities of the object functions. Finally, three examples are given to show the feasibility of the present approach.
\end{abstract}

\section{Introduction}

An urgent and realistic need in designing structures, e.g., car bodies, is to find an optimal design for minimizing vibration and noise, maximizing safety, minimizing the cost of products, etc. The need is constantly enforced in the process of contemporary commodity competition. Thus, structural optimization techniques have been developed rapidly to deal with these issues in recent years (e.g., Olhoff and Taylor 1983; Haftka and Grandhi 1986, 1992).

The simplest idea for optimizing a structure is to modify the sizes of structural members, i.e., use size parameters (plate thicknesses, bar cross-sectional areas, etc.) as design variables in the optimization process. This approach is called the sizing optimization method. Using the sizing optimization method one can improve the design of a structure in order to reduce vibration and noise, etc. But, if a structure was well designed by an experienced designer, then just limited improvement may be obtained by changing sizes. It has never been guaranteed that the sizing optimization can yield a truly optimal structure because the shape and topology of the structure are, in general, not modified (Bendsøe and Kikuchi 1988; Olhoff et al. 1991).

To attain the optimum, shape change of the structure has been considered by using boundary variation techniques (see, e.g., Haftka and Grandhi 1986). However, this method has some difficulties in practice, and still can seldomn yield a truly optimal structure. In the shape optimization, the initial topology of the structure must be set up by designers, which strongly depends on the experience of the designer, and it is very difficult to change the original topology during the optimization process. For example, if a singly connected domain is assumed at the initial design, the final structure obtained by a usual shape optimization method will remain the same topology as the initial one, although the truly optimal structure may have holes in the domain.

Little work had been done on optimization of the topology of a continuum structure before Bendsøe and Kikuchi (1988) despite extensive work on optimum topology of discrete structures such as trusses and grid-type structures (e.g., Kirsch 1989; Rozvany 1992). For finding optimum 
topology of a continuum structure, Bendsøe and Kikuchi (1988) applied a simple method of transforming the problem to find the Optimal Material Distribution (OMD) within a specified design domain from the original topology optimization problem. It is assumed that the material is not homogeneous, but instead has a variable solid-cavity microstructure. By using the homogenization method and a traditional Optimality Criteria (OC) updating algorithm, the optimal distribution of the material with respect to given loads and boundary conditions, were obtained. Their method provides very close results to the truly optimal structure.

This idea has opened up a new technique in structural optimization, and it has been successfully applied to find the optimum layout of a linearly elastic structure for its global stiffness (Bendsøe and Kikuchi 1988; Bendsøe 1989; Olhoff et al. 1991; Suzuki and Kikuchi 1990, 1991; Bendsøe et al. 1992). It is clear that applying this method to dynamic problems is also an important subject that has not been significantly expored. In fact, the dynamic problem is more important than the static one in design practice, e.g., in a car body design. Because of the difference of the dynamic problem from the static one, we must investigate what kind of dynamic optimization problems can be solved by the same method for stiffness and what kind of new techniques must be introduced to do so. In the stiffness optimization problem, the objective was minimization of the meancompliance of a given structure. But in optimization of vibrating structures, it is necessary to consider several different problems: the eigenvalue problem, frequency response problem, transient response problem, and others. These different problems may require different solution techniques, and solutions may be of very different natures.

The technique used in the stiffness problem has been applied to solve an eigenvalue optimization problem to find the optimal reinforcement of a plate-like structure (Diaz and Kikuchi 1992). However, the problem treated there is a special problem that the previous technique, the OC algorithm, could be applied to without much difficulty. Since practical design requires dealing with other type of dynamic optimization problems, we must examine the existing method to see whether it can be extended. In this paper, we shall consider the Frequency Response Optimization problem (FOR), and shall develop a new OC method.

Even though the existing Optimality Criteria (OC) method is well-convergent in the stiffness problem which uses the mean-compliance as the objective function (Bendsøe and Kikuchi 1988; Suzuki and Kikuchi 1990,1991), it does not work well in the frequency response problem, especially when the excitation frequency becomes high. A reason for this is that in the static problem, the sensitivities of the objective function are almost always negative (which means that increasing the design variables will reduce the mean-compliance). But in the frequency response optimization problem, some sensitivities of the objective function may be positive. In this case, the updated design variables can not be determined as a positive number by the previous OC updating rule that has been used so far. Although one can escape from this problem by giving the artificial values to the design variables, e.g., using some side constraints, it could cause a discontinuous jumping in the design variable, and there is no guarantee that the optimization process is going to converge to the optimum solution. This difficulty likely can be resolved by using a conventional Mathematical Programming method (MP), but the use of a conventional MP may result in very poor efficiency in our problem which involves a huge number of design variables. A major goal of this paper is to develop an efficient optimization algorithm. To this end, a Modified Optimality Criteria algorithm (MOC) is proposed.

\section{Formulation of the OMD problem}

A minimum weight (i.e., minimum amount of the material) problem which is subject to a constraint on the specified stiffness can be transformed to a dual problem that maximizes the stiffness subject to a constraint on the specified amount of the material. In general, a structural optimization problem can be essentially conceived as a rational search for the optimal spatial distribution of material within a prescribed admissible structural domain assuming the loading and boundary conditions to be given. This kind of problem is called Optimal Material Distribution (OMD) problem, and has been formulated by Bendsøe and Kikuchi (1988) using the concept of micro- 


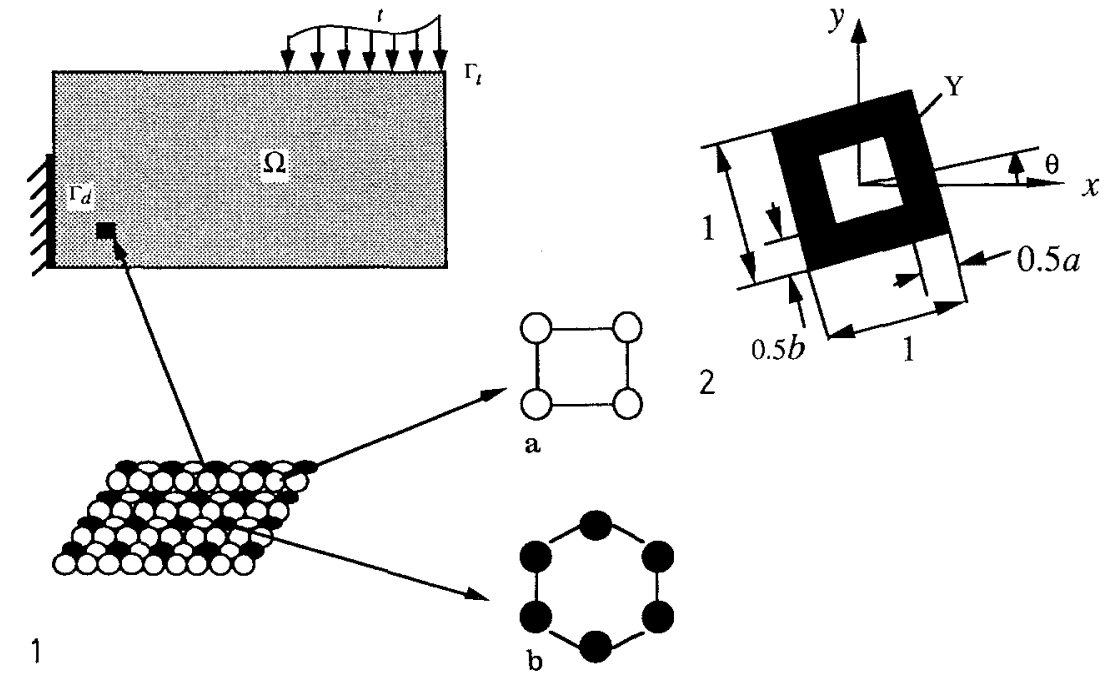

Figs. 1 and 2. 1 Microstructure of a structure, a void structure, b solid microstructure. 2 A special microstructure

structure for a structural stiffness problem. As shown in Fig. 1, it is considered that the structural domain is filled by a non-homogeneous material that has variable microstructures, and the empty cavity is also considered as a microstructure in which the mass and stiffness are zero. In the optimization process, the microstructures are changed form empty to solid or from solid to empty. Therefore, if one assumes that the total amount of the material of the structure is constant, then the pieces of the material are moved from a part of the structure to another part while the optimization process is progressed. By moving the material for reduction of the objective function, finally, we can obtain an OMD that gives the optimal structure.

There are infinitely many microstructures which can be considered for defining the problem. To simplify the problem, in the plane-stress problem, we assume that the microstructure is formed inside an empty rectangle in the so-called unit cell as shown in Fig. 2, where, $a, b$ and $\theta$ are regarded as the design variables for the OMD problem (Suzuki and Kikuchi 1990). The inside cavity of the microstructure is variable along with the design variables $a$ and $b$. And, the microstructure becomes a complete void when $a=b=0$ and a complete solid when $a=b=1$. The orientation $\theta$ stands for the rotation of the micro cell from a given fixed $x-y$ coordinate system as shown in Fig. 2 . Then the linear elasticity tensor $E_{i j k l}$ and the mass density $\rho$ become functions of these three design variables.

As noted earlier, there are many other ways to define microstructure with solid/cavity constituents. As well-know already (see, e.g., Bendsøe et al. 1992), the microstructure that yields the so-called rank 2 material might be a better choice to attain the maximum stiffness, but its optimum structural configuration tends to have a large amount of microscale cavities and is more porous. In other words, it is difficult to identify a discrete structure as well as topology of the structure using the rank 2 material model. On the other hand, the choice made here with a rectangular cavity in the unit cell results a clearer optimum structure, which can be easily identified. The choice of microstructure for OMD should be further investigated, but we shall not address this issue in the present paper.

Assuming $\Omega\left(\Omega \subset R^{3}\right)$ to be the structural possibly performed porous domain, $\Gamma_{t}$ the traction boundary of $\Omega$, equilibrium of such a structure with microstructure can be formulated by the weak form (the principle of virtual displacement):

$\int_{\Omega} E_{i j k l}^{\varepsilon} \frac{\partial u_{i}^{\varepsilon}}{\partial x_{j}} \frac{\partial v_{k}}{\partial x_{l}} d \Omega+\int_{\Omega} \rho^{\varepsilon} \frac{\partial^{2} u_{i}^{\varepsilon}}{\partial t^{2}} v_{i} d \Omega=\int_{\Omega} f_{i}^{\varepsilon} v_{i} d \Omega+\int_{\Gamma_{t}} t_{i} v_{i} d \Gamma \quad \forall \mathbf{v} \in V$

where,

$E_{i j k l}^{\varepsilon}=\left\{\begin{array}{ll}E_{i j k l}^{0} & \text { in solid } \\ 0 & \text { in cavity }\end{array}, \quad \rho^{\varepsilon}= \begin{cases}\rho_{0} & \text { in solid } \\ 0 & \text { in cavity }\end{cases}\right.$ 
$u_{i}^{\varepsilon}$ is the displacement that yields equilibrium, $v_{i}$ the virtual displacement $\left(\mathbf{v}=\left\{v_{1}, v_{2}, v_{3}\right\}^{T}\right), f_{i}^{\varepsilon}$ the body force, $t_{i}$ the boundary traction, $E_{i j k l}^{0}$ and $\rho_{0}$ are the elasticity tensor and mass density of the solid portion in $\Omega$, respectively. $V=\left\{\mathbf{v} \in H^{1}(\Omega)|\mathbf{v}|_{\Gamma_{d}}=0\right\}$ stands for the space of kinematically admissible displacement fields, and $\Gamma_{d}$ the displacement boundary, where $H^{1}(\Omega)$ is the Sobolev space in which the strain energy of the structure is finite. Here, the stress-strain and straindisplacement relations are assumed as

$\sigma_{i j}^{\varepsilon}=E_{i j k l}^{\varepsilon} e_{k l}^{\varepsilon}, \quad e_{k l}^{\varepsilon}=\frac{1}{2}\left(\frac{\partial u_{k}^{\varepsilon}}{\partial x_{l}}+\frac{\partial u_{l}^{\varepsilon}}{\partial x_{k}}\right)$.

Since $E_{i j k l}^{\varepsilon}$ and $\rho^{\varepsilon}$ are not homogeneous in $\Omega$, Eq. (1) has to be solved at both macroscopic and microscopic levels. Employing the homogenization method (see, e.g., Sanchez-Plencia 1980), Eq. (1) can be homogenized as

$\int_{\Omega} E_{i j k l}^{h} \frac{\partial u_{i}^{0}}{\partial x_{j}} \frac{\partial v_{k}}{\partial x_{l}} d \Omega+\int_{\Omega} \rho^{h} \frac{\partial^{2} u_{i}^{0}}{\partial t^{2}} v_{i} d \Omega=\int_{\Omega} f_{i}^{h} v_{i} d \Omega+\int_{\Gamma_{t}} t_{i} v_{i} d \Gamma$

where, $u_{i}^{0}$ stands for the component of the average displacements in the microstructural domain, $E_{i j k l}^{h}, \rho^{h}$ and $f_{i}^{h}$ the homogenized elastic coefficients, mass density and body force, respectively:

$E_{i j k l}^{h}=\frac{1}{|Y|_{Y}} \int_{Y}\left(E_{i j k l}^{\varepsilon}-E_{i j p q}^{\varepsilon} \frac{\partial \chi_{p}^{k l}}{\partial y_{q}}\right) d \mathbf{y}, \quad \rho^{h}=\frac{1}{|Y|_{Y}} \int_{Y} \rho^{\varepsilon} d \mathbf{y}, \quad f^{h}=\frac{1}{|Y|} \int_{Y} f^{\varepsilon} d \mathbf{y}$

where $Y$ stands for the domain of the microstructure, $|Y|$ the volume (area) of $Y$, and $\chi_{p}^{k l}$ is the solution of the microscopic problem that characterizes the micromechanical behavior of a specific microstructure:

$\int_{Y}\left(E_{i j k l}^{\varepsilon}-E_{i j p q}^{\varepsilon} \frac{\partial \chi_{p}^{k l}}{\partial y_{q}}\right) \frac{\partial v_{i}}{\partial y_{j}} d \mathbf{y}=0$ for $\forall \mathbf{v} \in V_{Y}$

where, space $V_{Y}=\left\{\mathbf{v}(\mathbf{y}) \in H^{1}(Y) \mid \mathbf{v}(\mathbf{y})\right.$ is $Y$-periodic $\}$ is defined on the microstructural domain $Y$.

The finite element method will be applied to solve the problem (5). Assuming the system is damped with viscous damping, the fundamental finite-element equation can be written by

$\mathbf{M u ̈}+\mathbf{C u}+\mathbf{K u}=\mathbf{f}$

where $\mathbf{u}, \mathbf{M}, \mathbf{C}, \mathbf{K}$ and $\mathbf{f}$ stand for the generalized displacement vector, mass matrix, damping matrix, stiffness matrix and generalized force vector, respectively. Here we assume the damping matrix is expressed by a linear combination of $\mathbf{K}$ and $\mathbf{M}$, i.e., $\mathbf{C}=\alpha \mathbf{K}+\beta \mathbf{M}$ for the given parameter $\alpha$ and $\beta$. It is noted that

$\mathbf{K}=\stackrel{n}{e l}_{e=1}^{n_{e l}} \mathbf{k}_{e}, \quad \mathbf{M}={\stackrel{n_{e l}}{A}}_{e=1}^{\mathbf{m}_{e}}$

where $\underset{e=1}{n_{e l}}$ stands for the finite element assembly operator, $\mathbf{k}_{e}$ and $\mathbf{m}_{e}$ the stiffness matrix and the mass matrix, which are obtained by

$\mathbf{k}_{e}=\int_{\boldsymbol{\Omega}_{e}} \mathbf{B}_{e}^{T} \mathbf{D}_{e} \mathbf{B}_{e} d \Omega, \quad \mathbf{m}_{e}=\int_{\Omega_{e}} \rho_{e} \mathbf{N}_{e}^{T} \mathbf{N}_{e} d \Omega$

respectively. Where, $\mathbf{D}_{e}=\mathbf{T}_{e}^{h} \mathbf{D}^{h} \mathbf{T}_{e}$ stands for the rotated homogenized stiffness matrix, $\rho_{e}=\rho^{h}$. In the plane stress problem using the microstructure shown in Fig. 2, we have

$$
\mathbf{D}^{h}=\left[\begin{array}{ccc}
E_{1111}^{h} & E_{1122}^{h} & 0 \\
E_{1122}^{h} & E_{2222}^{h} & 0 \\
0 & 0 & E_{1212}^{h}
\end{array}\right], \quad \mathbf{T}_{e}=\left[\begin{array}{ccc}
\cos ^{2} \theta & \sin ^{2} \theta & \cos \theta \sin \theta \\
\sin ^{2} \theta & \cos ^{2} \theta & -\cos \theta \sin \theta \\
-2 \cos \theta \sin \theta & 2 \cos \theta \sin \theta & \cos ^{2} \theta-\sin ^{2} \theta
\end{array}\right] .
$$


It is assumed that, in a finite-element, all microstructures have the same sizes and orientation. Thus, the design variables $a, b$ and $\theta$ can be discretely defined in each finite-element using the symbols $a_{e}, b_{e}$ and $\theta_{e}$. Where, the subscript $e$ implies that the design variables $a_{e}, b_{e}$ and $\theta_{e}$ are for a finite-element $\Omega_{e}$. Since $\mathbf{D}_{e}$ and $\boldsymbol{\rho}_{e}$ are the functions of the discrete design variables, $a_{e}, b_{e}$ and $\theta_{e}$, the elementary stiffness and mass matrices are the functions of the design variables, i.e.,

$\mathbf{k}_{e}=\mathbf{k}_{e}\left(a_{e}, b_{e}, \theta_{e}\right), \quad \mathbf{m}_{e}=\mathbf{m}_{e}\left(a_{e}, b_{e}\right)$.

For the frequency response problem, assuming $\mathbf{f}=\mathbf{F} e^{j \omega t}$ and $\mathbf{u}=\mathbf{U} e^{j \omega t}$, we have

$\left(\mathbf{K}+j \omega \mathbf{C}-\omega^{2} \mathbf{M}\right) \mathbf{U}=\mathbf{F}$

where $j=\sqrt{-1}$.

\section{Structural optimization problems for frequency response}

\subsection{Objective functions}

Many kinds of objective functions can be considered for the optimization problem. For example, minimizing the frequency response in some specified nodes of the structure, minimizing the strain or kinetic energy of the structure with respect to a given frequency, are two of many possibilities. In the static problem (Bendsøe and Kikuchi 1988; Bendsøe 1989; Olhoff et al. 1991; Suzuki and Kikuchi 1990, 1991; Bendsøe et al. 1992), the mean-compliance, i.e., $f=\mathbf{u}^{T} \mathbf{f}$, is considered as the objective function in the optimization problem. In this paper we shall extend this objective function to the frequency response problem. The following two problems are specially considered:

i) Find the stiffest structure against the specified exciting frequency or the exciting frequencies in a frequency domain for a fixed, given volume of material.

ii) Reduce the frequency response over whole structure with respect to the given exciting frequency or a frequency domain.

Thus, we consider the following two objective functions:

a) Minimizing the absolute value (modulus) of the mean-compliance of the structure

$f_{1}=\left|\mathbf{U}^{T} \mathbf{F}\right|$

here, the generalized mean-compliance $\mathbf{U}^{T} \mathbf{F}$ is a function of the exciting frequency $\omega$, and, in the general case, is a complex number.

b) Minimizing the integrates of the object function defined above within a certain frequency domain $\left[\omega_{a}, \omega_{b}\right]$.

$f_{2}=\int_{\omega_{a}}^{\omega_{b}} f_{1}(\omega) d \omega$

Note that Eq. (18) might be approximated by quadrature

$f_{2}=\sum_{i} w_{i} f_{i}\left(\omega_{i}\right)$

where $\left\{w_{i}, \omega_{i}\right\}$ is the set of quadrature weights and points.

\subsection{Constraints}

Consider a constraint of total mass of the structure:

$\int_{\Omega} \rho_{e} d \Omega \leqq W_{0}$ 
where, $W_{0}$ stands for the given total mass of the structure. In the problem with the microstructure shown in Fig. 2, $\rho_{e}=\rho_{0}\left(a_{e}+b_{e}-a_{e} b_{e}\right)$, therefore the constraint function becomes

$h=\sum_{e=1}^{n_{e l}} \rho_{0}\left(a_{e}+b_{e}-a_{e} b_{e}\right)-W_{0} \leqq 0$.

In general, the lower and upper bounds for the design variables $a_{e}$ and $b_{e}$ have to be specified:

$\underline{a}_{e} \leqq a_{e} \leqq \bar{a}_{e}, \quad \underline{b}_{e} \leqq b_{e} \leqq \bar{b}_{e} \quad\left(e=1,2, \ldots, n_{e l}\right)$

where, $\underline{a}_{e}\left(\underline{b}_{e}\right)$ and $\bar{a}_{e}\left(\bar{b}_{e}\right)$ stand for the permissible minimum and maximum values of the design variables $a_{e}\left(b_{e}\right)$, respectively. In the theoretical case, for the unit cell shown in Fig. 2, we have $\underline{a}_{e}=\underline{b}_{e}=0$ and $\bar{a}_{e}=\bar{b}_{e}=1\left(e=1,2, \ldots, n_{e l}\right)$. But, for convenience in practical implementation, we consider $\underline{a}_{e}=\underline{b}_{e}=\delta\left(e=1,2, \ldots, n_{e l}\right)$ for the lower bounds of the design variables instead of zero values, where $\delta$ is a sufficiently small number.

\subsection{General formulation of the optimization problem}

The optimization problems can be written as

Minimize $f(\mathbf{U}), \quad$ subject to $h(\mathbf{X}) \leqq 0, \quad \underline{x}_{i} \leqq x_{i} \leqq \bar{x}_{i}, \quad(i=1,2, \ldots, N)$

where, $\mathbf{X}=\operatorname{col}\left\{x_{i}\right\}\left(x_{i} \in A \cup B\right), \Theta=\operatorname{col}\left\{\theta_{i}\right\}$ are vectors of the design variables, $A$ and $B$ stand for the sets of the design variables $a_{i}$ and $b_{i}$, respectively; $\underline{x}_{i}$ and $\bar{x}_{i}$ are the minimum and maximum values of the design variables $x_{i}$, respectively; and $N=2 n_{e l}$. The object function $f$ can be any one of the objective functions that we defined previously. In addition, it is assumed that the state equation, Eq. (16), is always satisfied by solving a structural frequency response analysis problem.

\section{Optimization algorithm}

The Lagrangian function of the constrained optimization problem can be defined as

$L=f+\lambda h+\sum_{i=1}^{N}\left[\alpha_{-i}\left(\underline{x}_{i}-x_{i}\right)+\alpha_{+i}\left(x_{i}-\bar{x}_{i}\right)\right]$

where, $\lambda, \alpha_{-i}$ and $\alpha_{+i}$ are the Lagrangian multipliers. Then, the Kuhn-Tucker conditions of the problem become

$\frac{\partial f}{\partial x_{i}}+\lambda \frac{\partial h}{\partial x_{i}}=\alpha_{-i}-\alpha_{+i}, \quad(i=1,2, \ldots, N) ; \quad \frac{\partial f}{\partial \theta_{i}}=0, \quad\left(i=1,2, \ldots, n_{e l}\right)$

$\lambda h=0 ; \quad \alpha_{-i}\left(\underline{x}_{i}-x_{i}\right)=0, \quad \alpha_{+i}\left(x_{i}-\bar{x}_{i}\right)=0, \quad(i=1,2, \ldots, N)$

where, $\lambda \geqq 0, \alpha_{-i} \geqq 0, \alpha_{+i} \geqq 0(i=1,2, \ldots, N)$.

An iterative optimization algorithm employed by Bendsøe and Kikuchi (1988) and Suzuki and Kikuchi $(1990,1991)$, which was utilized for solving the stiffness optimization problem, can be written as follows: $k=0$.

a) Give the initial value of the design variables, $x_{i}^{k}(i=1,2, \ldots, N)$ and $\theta_{i}^{k}\left(i=1,2, \ldots, n_{e l}\right)$, where

b) Find the solution $\mathbf{U}^{k}$ of state equation Eq. (16) with respect to $x_{i}=x_{i}^{k}, \theta_{i}=\theta_{i}^{k}$

c) Calculate $\partial f / \partial x_{i}$ and $\partial h / \partial x_{i}$ with respect to $x_{i}=x_{i}^{k}, \theta_{i}=\theta_{i}^{k}$

d) Modify the design variables as follows:

$$
x_{i}^{k+1}= \begin{cases}x_{i}^{k} & \text { if } \quad x_{i}^{k} D_{i}^{k} \leqq x_{i}^{k} \\ x_{i}^{k} D_{i}^{k} & \text { if } \quad x_{i}^{k}<x_{i}^{k} D_{i}^{k}<\bar{x}_{i}^{k}, \quad(i=1,2, \ldots, N) \\ \bar{x}_{i}^{k} & \text { if } \quad x_{i}^{k} D_{i}^{k} \geqq \bar{x}_{i}^{k}\end{cases}
$$


where

$$
D_{i}^{k}=\left.\left[-\left(\frac{\partial h}{\partial x_{i}} \lambda^{k}\right)^{-1} \frac{\partial f}{\partial x_{i}}\right]^{\eta}\right|_{x_{i}=x_{i}^{k}, \theta_{i}=\theta_{i}^{k}} ; \quad \underline{x}_{i}^{k}=\max \left\{(1-\zeta) x_{i}^{k}, \underline{x}_{i}\right\}, \quad \bar{x}_{i}^{k}=\min \left\{(1+\zeta) x_{i}^{k}, \bar{x}_{i}\right\},
$$

e) Determine $\theta_{i}^{k+1}$ using Eq. (26) or its equivalent form (Suzuki and Kikuchi 1990).

f) Let $k=k+1$, and repeat b) to e) until some convergence criteria are fulfilled.

In above, $\eta$ and $\zeta$ are given, and are regarded as the step size parameters. The $k$-th approximation of the Lagrangian multiplier, $\lambda^{k}$, is adjusted by an inner iteration loop to satisfy the constraint $h\left(\mathbf{x}^{k}\left(\lambda^{k}\right)\right)=0$ by assuming this constraint is always active.

The updating rule Eq. (28) in this algorithm is based on the traditional Optimality Criteria (OC) method (e.g., Berke and Venkayya 1974). Even though this algorithm is convergent for most of the static problems of a linearly elastic structure (Bendsøe and Kikuchi 1988; Suzuki and Kikuchi 1990, 1991), it does not work well in the dynamic case, especially when exciting frequency becomes high. The reason for this is that in the static problem, the derivatives of the objective and constraint functions do not change their sign during the iteration of optimization, i.e.,

$\partial f / \partial x_{i} \leqq 0 \quad$ and $\quad \partial h / \partial x_{i} \geqq 0, \quad\left(\right.$ for $\forall x_{i} \in A \cup B, x_{i} \geqq 0$ ).

Thus, we can find a Lagrangian multiplier $\lambda^{k}>0$ and convert $D_{i}^{k}$ in Eq. (29) to be a positive real number, and then the design variables can be updated as positive real number via Eq. (28). However, in the dynamic problem, some derivatives of the object function may become positive. In the case that Eq. (31) is not satisfied, because $\lambda^{k}>0$ (Lagrangian multiplier has to be a positive number), the updated design variable $x_{i}^{k+1}$ calculated by Eq. (28) may be a negative or complex number. Even though this undesirable behavior can be avoided by letting the design variable to be some artificial value, e.g., $x_{i}^{k+1}=\underline{x}_{i}^{k}$ (when $\partial f / \partial x_{i}>0$ and $\partial h / \partial x_{i} \geqq 0$ ), where $\underline{x}_{i}^{k}$ is a moving limit as shown in Eq. (30); it could cause a discontinuous jump in the design variable, and it yields no guarantee for convergence of the optimization process to its local minima.

In this paper, we propose a new optimization algorithm to overcome the difficulty mentioned in above. The basic idea is the use of a convex approximation, which is the basis of widely used optimization methods CONLIN (Fleury and Braibant 1986) and MMA (Svanberg 1987). The method introduced here is different from the CONLIN and the MMA, and it employs a shift parameter which corresponds to the Lagrange multiplier to make the convex approximation of the problem. The new algorithm can be reduced to the traditional OC algorithm, and it has the same simplicity as the OC method.

Rewrite Eq. (25) as

$\left(\frac{\partial f}{\partial x_{i}}-\mu \frac{\partial h}{\partial x_{i}}\right)+\lambda^{*} \frac{\partial h}{\partial x_{i}}=\alpha_{-i}-\alpha_{+i}, \quad(i=1,2, \ldots, N)$

where, $\mu$ is a shift parameter, and $\lambda^{*}=\lambda+\mu$. If we assume Eq. (23b) to be active, but Eq. (23c) not active, then Eq. (32) becomes

$\frac{\partial f^{*}}{\partial x_{i}}+\lambda^{*} \frac{\partial h}{\partial x_{i}}=0, \quad(i=1,2, \ldots, N)$

where, $f^{*}=f-\mu h$. Now we have new optimization problem with the objective function $f^{*}$ and the constraint $h \leqq 0$. This problem is equivalent to the original one.

Using a generalized reciprocal approximation, we assume the intermediate variables for the linearization as

$y_{i}=\left(\frac{1}{x_{i}}\right)^{\xi}, \quad(i=1,2, \ldots, N)$

where $\xi>0$ is a given parameter. Assuming that $x_{i}^{k}(i=1,2, \ldots, N)$ are the $k$-th approximation of 
the optima obtained in last interaction step, $f^{*}$ can be linearly approximated in terms of $y_{i}$ at the point $y_{i}=y_{i}^{k}=\left(x_{i}^{k}\right)^{-\xi}(i=1,2, \ldots, N)$ as

$f^{k}=f_{0}^{k}+\sum_{i=1}^{N} a_{i}^{k} x_{i}^{-\xi}$

where $f_{0}^{k}$ is a constant, and

$a_{i}^{k}=\left.\frac{\partial f^{*}}{\partial y_{i}}\right|_{y_{i}=y_{i}^{k}}=-\frac{1}{\xi}\left(\frac{\partial f}{\partial x_{i}}-\mu \frac{\partial h}{\partial x_{i}}\right)_{x_{i}=x_{i}^{k}}\left(x_{i}^{k}\right)^{\xi+1}, \quad(i=1,2, \ldots, N)$.

If we choose the shift parameter $\mu$ in the $k$-th interaction step as

$\mu^{k} \geqq\left.\left[\frac{\partial f}{\partial x_{i}} / \frac{\partial h}{\partial x_{i}}\right]\right|_{x_{i}=x_{i}^{k}} \quad($ for $i=1,2, \ldots, N)$

then, from Eq. (36), we have $a_{i}^{k} \geqq 0(i=1,2, \ldots, N)$, and the approximate objective function $f^{k}$ is convex.

The constraint function $h$ is a bilinear function of the design variables in this problem, therefore it can be linearized in terms of the direct design variables $x_{i}$ 's as

$h^{k}=h_{0}^{k}+\sum_{i=1}^{N} b_{i}^{k} x_{i}$

where, $h_{0}^{k}<0$ is a constant, and

$b_{i}^{k}=\left.\frac{\partial h}{\partial x_{i}}\right|_{x_{i}=x_{i}^{k}}, \quad\left(b_{i}^{k} \geqq 0 ; i=1,2, \ldots, N\right)$.

Then the Lagrangian function in the approximated problem becomes

$L^{k}\left(\mathbf{X}, \lambda^{k}\right)=l_{0}^{k}+\sum_{i=1}^{N} a_{i}^{k} x_{i}^{-\xi}+\lambda^{k} \sum_{i=1}^{N} b_{i}^{k} x_{i}=l_{0}^{k}+\sum_{i=1}^{N} l_{i}^{k}$

where

$l_{0}^{k}=f_{0}^{k}+\lambda^{k} h_{0}^{k}, \quad l_{i}^{k}=a_{i}^{k} x_{i}^{-\xi}+\lambda^{k} b_{i}^{k} x$.

Since the approximated Lagrangian function $L^{k}\left(\mathbf{X}, \lambda^{k}\right)$ is convex for any $\lambda^{k} \geqq 0$, we can use the dual method to solve the problem (see, e.g., Haftka and Gurdal 1992). The dual problem is defined by

Maximize $L_{m}^{k}\left(\lambda^{k}\right), \quad$ subject to $\lambda^{k} \geqq 0$

where

$L_{m}^{k}\left(\lambda^{k}\right)=\min _{\underline{\mathbf{X}}<\mathbf{X}<\overline{\mathbf{X}}} L^{k}\left(\mathbf{X}, \lambda^{k}\right)$

Because the minimization problem Eq. (44) is separable, it can be replaced with $N$ onedimensional minimization problems of the form:

Minimize $l_{i}^{k}\left(x_{i}\right)=a_{i}^{k} x_{i}^{-\xi}+\lambda^{k} b_{i}^{k} x_{i}, \quad$ subject to $\underline{x}_{i}<x_{i}<\bar{x}_{i}$

where, $a_{i}^{k} \geqq 0, b_{i}^{k} \geqq 0,(i=1,2, \ldots, N)$. By solving this simple minimization problem, we can obtain the solution

$x_{i}^{*}=\left(\frac{\xi a_{i}^{k}}{\lambda^{k} b_{i}^{k}}\right)^{1 /(\xi+1)}, \quad\left(\underline{x}_{i}<x_{i}^{*}<\bar{x}_{i}, i=1,2, \ldots, N\right)$.

Using $x_{i}^{*}$ as the $(k+1)$-th approximation of the solution of the optimization problem, i.e., 
$x_{i}^{k+1}=x_{i}^{*}$, and substituting Eqs. (36) and (39) into Eq. (46), we have

$$
x_{i}^{k+1}=\left[\frac{1}{\lambda^{k}}\left(\mu^{k}-\left.\left(\frac{\partial f / \partial x_{i}}{\partial h / \partial x_{i}}\right)\right|_{x_{i}=x_{i}^{k}}\right)\right]^{\eta} x_{i}^{k} \quad\left(\underline{x}_{i}<x_{i}^{k+1}<\bar{x}_{i}, i=1,2, \ldots, N\right)
$$

where $\eta=1 /(\xi+1)$, and

$$
\mu^{k} \geqq \max _{1 \leqq i \leqq N}\left\{\left.\left(\frac{\partial f / \partial x_{i}}{\partial h / \partial x_{i}}\right)\right|_{x_{i}=x_{i}^{k}}\right\} \text {. }
$$

The solution Eq. (46) can be rewritten as

$$
x_{i}^{k+1}=\left(\lambda^{k}\right)^{-\eta} e_{i}^{k}, \quad \text { where } \quad e_{i}^{k}=\left(\frac{\xi a_{i}^{k}}{b_{i}^{k}}\right)^{\eta} \text {. }
$$

Substituting Eq. (49) into Eq. (40) gives

$$
L^{k}=f_{0}^{k}+\lambda^{k} h_{0}^{k}+\left(\lambda^{k}\right)^{1-\eta} \sum_{i=1}^{N}\left[a_{i}^{k}\left(e_{i}^{k}\right)^{-\xi}+b_{i}^{k} e_{i}^{k}\right]
$$

Because

$a_{i}\left(e_{i}^{k}\right)^{-\xi}=\frac{1}{\xi} b_{i}^{k} e_{i}^{k}, \quad$ and $\quad \frac{1}{\xi}+1=\frac{1}{1-\eta}$

substituting Eq. (51) into Eq. (50) yields

$$
L^{k}=f_{0}^{k}+\lambda^{k} h_{0}^{k}+\left(\lambda^{k}\right)^{1-\eta}(1-\eta)^{-1} \sum_{i=1}^{N} b_{i}^{k} e_{i}^{k} .
$$

To solve the maximization problem Eq. (43), we have

$$
\frac{\partial L^{k}}{\partial \lambda}=h_{0}^{k}+\left(\lambda^{k}\right)^{-\eta} \sum_{i=1}^{N} b_{i}^{k} e_{i}^{k}=0 .
$$

Then the Lagrangian multiplier can be obtained as

$$
\lambda^{k}=\left(\frac{-1}{h_{0}^{k}} \sum_{i=1}^{N} b_{i}^{k} e_{i}^{k}\right)^{1 / \eta}
$$

where $\lambda^{k} \geqq 0\left(h_{0}^{k}<0\right)$.

Equation (54) has to be revised if some side constraints for the lower and upper bounds of the design variables in Eq. (23c) are active. Assuming $I_{-}^{k}$ and $I_{+}^{k}$ are the sets of numbers of the design variables which arrive the minimum and maximum values respectively, and $I^{k}$ is the set of numbers of the other design variables. Then the Lagrangian function in the $k$-th iteration, Eq. (40), can be revised as

$$
L^{k}\left(\mathbf{X}, \lambda^{k}\right)=l_{0}^{k}+\sum_{i \in I^{k}} l_{i}^{k}+\sum_{i \in I_{-}^{k}} l_{-i}^{k}+\sum_{i \in I_{+}^{k}} l_{+i}^{k}
$$

where

$$
\begin{aligned}
& l_{0}^{k}=f_{0}^{k}+\lambda^{k} h_{0}^{k}+\sum_{i \in I_{-}^{k}} \alpha_{-i} \underline{x}_{i}-\sum_{i \in I_{+}^{k}} \alpha_{+i} \bar{x}_{i} \\
& l_{i}^{k}=a_{i}^{k} x_{i}^{-\xi}+\lambda^{k} b_{i}^{k} x_{i}, \quad l_{i}^{k}=a_{i}^{k} x_{i}^{-\xi}+\lambda^{k} b_{i}^{k} x_{i}, \quad l_{+i}^{k}=a_{i}^{k} x_{i}^{-\xi}+\lambda^{k} b_{i}^{k} x_{i}+\alpha_{+i} x_{i} .
\end{aligned}
$$

By solving the dual problem Eq. (43) with respect to the Lagrangian function defined by Eq. (55), we can obtain

$$
\begin{aligned}
& x_{i}^{k+1}=\left(\lambda^{k}\right)^{-\eta} e_{i}^{k}, \quad\left(\text { for } \underline{x}_{i} \leqq\left(\lambda^{k}\right)^{-\eta} e_{i}^{k} \leqq \bar{x}_{i}, i=1,2, \ldots, N\right) \\
& \alpha_{-i}=b_{i}^{k}\left[\lambda^{k}-\left(e_{i}^{k} / \underline{x}_{i}\right)^{1 / \eta}\right], \quad\left(\alpha_{-i} \geqq 0\right), \quad \alpha_{+i}=b_{i}^{k}\left[\left(e_{i}^{k} / \bar{x}_{i}\right)^{1 / \eta}-\lambda^{k}\right], \quad\left(\alpha_{+i} \geqq 0\right)
\end{aligned}
$$


$\lambda^{k}=\left(\frac{-1}{\tilde{h}_{0}^{k}} \sum_{i \in I^{k}} b_{i}^{k} e_{i}^{k}\right)^{1 / \eta}, \quad$ where $\quad \tilde{h}_{0}^{k}=h_{0}^{k}+\sum_{i \in I_{-}^{k}} b_{i}^{k} \underline{x}_{i}+\sum_{i \in I_{+}^{k}} b_{i}^{k} \bar{x}_{i}$.

It should be noted that because the sets $I_{-}^{k}, I^{k}$ and $I_{+}^{k}$ depend on the solution of Lagrangian multiplier $\lambda^{k}$, an iterative calculations is required for solving $\lambda^{k}$.

In summary, the improved optimization algorithm can be described by $k=0$.

a) Give the initial value of the design variables, $x_{i}^{k}(k=1,2, \ldots, N)$ and $\theta_{i}^{k}\left(i=1,2, \ldots, n_{e l}\right)$, where

b) Find the solution $\mathbf{U}^{k}$ of governing equation Eq. (16) with respect to $x_{i}=x_{i}^{k}, \theta_{i}=\theta_{i}^{k}$.

c) Calculate $\partial f / \partial x_{i}$ and $\partial h / \partial x_{i}$ with respect to $x_{i}=x_{i}^{k}, \theta_{i}=\theta_{i}^{k}$.

d) Calculate $\mu^{k}$ using Eq. (48)

e) Calculate

$e_{i}^{k}=\hat{D}_{i}^{k} x_{i}^{k}, \quad(i=1,2, \ldots, N), \quad$ where, $\quad \hat{D}_{i}^{k}=\left(\mu^{k}-\left.\left(\frac{\partial f / \partial x_{i}}{\partial h / \partial x_{i}}\right)\right|_{x_{i}=x_{i}^{k}}\right)^{\eta}$.

f) Determine the Lagrangian multiplier by an inner iteration loop using

$\tilde{\lambda}^{k}=\frac{-1}{\tilde{h}_{0}^{k}} \sum_{i \in I^{k}} b_{i} e_{i}^{k}, \quad$ where $\tilde{\lambda}^{k}=\left(\lambda^{k}\right)^{\eta}, \quad \tilde{h}_{0}^{k}=h_{0}^{k}+\sum_{i \in I_{-}^{k}} b_{i}^{k} \underline{x}_{i}+\sum_{i \in I_{+}^{k}} b_{i}^{k} \bar{x}_{i}$,

$I_{-}^{k}=\left\{i \mid e_{i}^{k} / \tilde{\lambda}^{k} \leqq \underline{x}_{i}\right\}, \quad I^{k}=\left\{i \mid \underline{x}_{i}<e_{i}^{k} / \tilde{\lambda}^{k}<\bar{x}_{i}\right\}, \quad I_{+}^{k}=\left\{i \mid e_{i}^{k} / \tilde{\lambda}^{k} \geqq \bar{x}_{i}\right\}$

g) Modify the design variables as follows:

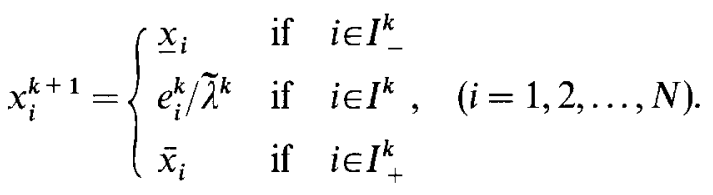

h) Determine $\theta_{i}^{k+1}$ using Eq. (26) or its equivalent form (Suzuki and Kikuchi 1990).

i) Let $k=k+1$, and repeat b) to h) until some convergence criteria are fulfilled.

Note that if

$\left.\frac{\partial f}{\partial x_{i}}\right|_{x_{i}=x_{i}^{k}} \leqq 0$ for $\forall x_{i}^{k} \in A \cup B\left(\underline{x}_{i} \leqq x_{i}^{k} \leqq \bar{x}_{i}\right)$

is satisfied, we can choose the shift parameter as $\mu^{k}=0$, then the updating rule Eq. (67) is reduced to the previous OC updating rule employed by Bendsøe and Kikuchi (1988), i.e., Eq. (28), which is used for the static stiffness problems. Therefore, the new optimization algorithm obtained here is a generalization of the previous one, which can be applied to more general problems. It should be noted that the calculation of the Lagrange multiplier has also been significantly improved. In the previous method, the Lagrange multiplier is calculated by solving a nonlinear equation using, e.g., the bi-section method (Suzuki and Kikuchi 1990). But, in the new algorithm, using Eq. (64), the Lagrange multiplier can be obtained through only two or three iterations with much simpler calculation. Therefore, the optimization algorithm presented here is more efficient than the previous one.

As well known in the OC method, the step size parameter $\eta$ in Eqs. (29) and (63) plays very important rule in the optimization process. A large value of $\eta$ can accelerate the convergence toward to the final solution, but it may cause oscillation in the iterative process. A small value of $\eta$ can prevent divergence, but it results in small change to the design variables, which slows down the progress of convergence [e.g., 12]. However, the OC approach could not give a theoretical guideline about what is the optimal value of the parameter $\eta$. In this paper, we can see, $\eta$ is related to the parameter $\xi$, i.e., $\eta=1 /(\xi+1)$, which is the exponent of the reciprocal variables used in the approximation process (see Eq. (34)). Therefore, the optimal value of $\eta$ is dependent on the optimal value of the parameter $\xi$. It is clear that if the approximate objective function, i.e., Eq. (35), is closer 
to the primary one, the optimal solution of the approximate optimization problem will be closer to the solution of the primary problem. Then less iterations will be needed for obtaining the optima of the primary problem. Therefore, the optimal $\xi$ is that which can make the approximate objective function obtained using the intermediate variables $y_{i}$ of Eq. (34) to be as close to the primary one as possible.

\section{Derivative calculations of the object functions}

The major computation in the aforementioned problem is the calculation of the derivatives of the objective functions, $\partial f_{i} / \partial x_{k}$, where $x_{k}$ stands for an arbitrary design variable. Two types of sensitivity analysis methods have been proposed by Ma and Hagiwara (1991a, 1992) for a frequency response problem. One is the Direct Frequency Response (DRF) method (Ma and Hagiwara 1991a), the other is the Modal Frequency Response (MFR) method (Ma and Hagiwara 1992). In this section, we shall apply these methods to the optimization problems defined in this paper. For the sake of simplicity, the damping matrix is omitted in the following sections.

\subsection{Derivatives via the DFR method}

Performing a partial derivative operation on Eq. (16) for the case of no damping, $\mathbf{C}=\mathbf{0}$, with respect to design variable $x_{k}$, results in

$\left(\frac{\partial \mathbf{K}}{\partial x_{k}}-\omega^{2} \frac{\partial \mathbf{M}}{\partial x_{k}}\right) \mathbf{U}+\left(\mathbf{K}-\omega^{2} \mathbf{M}\right) \frac{\partial \mathbf{U}}{\partial x_{k}}=0$.

Suppose that the exciting frequency $\omega$ is not equal to any natural frequency of the structure. Then the derivative of the frequency response can be obtained as

$\frac{\partial \mathbf{U}}{\partial x_{k}}=-\left(\mathbf{K}-\omega^{2} \mathbf{M}\right)^{-1}\left(\frac{\partial \mathbf{K}}{\partial x_{k}}-\omega^{2} \frac{\partial \mathbf{M}}{\partial x_{k}}\right) \mathbf{U}$.

Using Eq. (70) and $\mathbf{U}=\left(\mathbf{K}-\omega^{2} \mathbf{M}\right)^{-1} \mathbf{F}$, we can obtain the sensitivity of the object function $f_{1}$, which is defined in Eq. (17), as

$\frac{\partial f_{1}}{\partial x_{k}}=-\operatorname{sign}\left\{f_{1}\right\} \mathbf{U}^{T}\left(\frac{\partial \mathbf{K}}{\partial x_{k}}-\omega^{2} \frac{\partial \mathbf{M}}{\partial x_{k}}\right) \mathbf{U}$.

Because the design variable $x_{k}$ belongs to the $k$-th element only, using Eq. (11), the above equation can be greatly simplified as

$\frac{\partial f_{1}}{\partial x_{k}}=-\operatorname{sign}\left\{f_{1}\right\} \mathbf{U}_{k}^{T}\left(\frac{\partial \mathbf{k}_{k}}{\partial x_{k}}-\omega^{2} \frac{\partial \mathbf{m}_{k}}{\partial x_{k}}\right) \mathbf{U}_{k}$.

where, $\mathbf{U}_{k}$ stands for the component of $\mathbf{U}$ which corresponds to the $k$-th finite-element $\Omega_{k}$ only, and $\partial \mathbf{k}_{k} / \partial x_{k}$ and $\partial \mathbf{m}_{k} / \partial x_{k}$ are calculated by

$\frac{\partial \mathbf{k}_{k}}{\partial x_{k}}=\int_{\Omega_{k}} \mathbf{B}_{k}^{T} \frac{\partial \mathbf{D}_{k}}{\partial x_{k}} \mathbf{B}_{k} d \Omega, \quad \frac{\partial \mathbf{m}_{k}}{\partial x_{k}}=\int_{\Omega_{k}} \frac{\partial \rho_{k}}{\partial x_{k}} \mathbf{N}_{k}^{T} \mathbf{N}_{k} d \Omega$.

Note that Eq. (72) only needs to be calculated at the element level. For example, if we use 4-node quadrilateral elements, just 72 multiplications are needed for each derivative calculation with respect to an individual design variable. Therefore, the derivative calculation using the method proposed here is very simple and highly efficient, especially compared with the use of a finite-difference method.

It should also be noted that the derivatives obtained using Eq. (72) are dependent on the given exciting frequency $\omega$. Especially, in Eq. (72), the component of the frequency response, $\mathbf{U}_{k}$, is an 
implicit function of $\omega$, and it is obtained by solving the state equation Eq. (16). Thus, for a different value of $\omega$, the Eq. (16) has to be completely reevaluated. Therefore, it becomes very inefficient if we need to calculate the derivatives which are related to many frequency points or a frequency domain, e.g., in the case of objective function $f_{2}$, because solving Eq. (16) is the most expensive procedure in the problem. Therefore, it is necessary to introduce the Modal Frequency Response (MFR) sensitivity analysis method (Ma and Hagiwara 1992) for the problem.

\subsection{Derivatives via the MFR method}

Using a standard mode-superposition technique (mode-displacement method), the frequency response $\mathbf{U}$ can be expressed as

$\mathbf{U}=\sum_{i=1}^{n} \phi_{i} q_{i}, \quad q_{i}=\frac{\phi_{i}^{T} \mathbf{F}}{\omega_{i}^{2}-\omega^{2}}$

where $\omega_{i}^{2}$ and $\phi_{i}$ stand for the $i$-the eigenvalue and eigenvector of the structure, respectively. Eq. (74) can be also written as a matrix form:

$\mathbf{U}=\Phi \mathbf{Q}$

where $\Phi=\left[\phi_{1}, \phi_{2}, \ldots, \phi_{n}\right]$ and $\mathbf{Q}=\operatorname{col}\left\{q_{i}\right\}$. Then the derivatives of the MFR in terms of modal coordinates can be obtained as following.

$\frac{\partial \mathbf{U}}{\partial x_{k}}=\Phi \mathbf{S Q}$

Here, $\mathbf{S}=\left(S_{i j}\right)$ is called sensitivity matrix, and

$S_{i j}=\frac{-1}{\omega_{i}^{2}-\omega^{2}} \phi_{i k}^{T}\left(\frac{\partial \mathbf{k}_{k}}{\partial x_{k}}-\omega^{2} \frac{\partial \mathbf{m}_{k}}{\partial x_{k}}\right) \phi_{j k}=\frac{-1}{\omega_{i}^{2}-\omega^{2}}\left(k_{k i j}^{*}-\omega^{2} m_{k i j}^{*}\right), \quad(i, j=1,2, \ldots, n)$

where $\phi_{i k}$ stands for the component of $\phi_{i}$ with respect to the $k$-th finite-element of the structure, and

$k_{k i j}^{*}=\phi_{i k}^{T} \frac{\partial \mathbf{k}_{k}}{\partial x_{k}} \phi_{j k}, \quad m_{k i j}^{*}=\phi_{i k}^{T} \frac{\partial \mathbf{m}_{k}}{\partial x_{k}} \phi_{j k}, \quad(i, j=1,2, \ldots, n)$

are independent from the exciting frequency $\omega$.

Therefore, the derivatives of the objective functions defined in this paper can be obtained as:

$\frac{\partial f_{1}}{\partial x_{k}}=\operatorname{sign}\left\{f_{1}\right\} \mathbf{Q}^{T} \mathbf{S}^{1} \mathbf{Q}$ and $\frac{\partial f_{2}}{\partial x_{k}}=\int_{\omega_{a}}^{\omega_{b}} \operatorname{sign}\left\{f_{1}\right\} \mathbf{Q}^{T} \mathbf{S}^{1} \mathbf{Q} d \omega$

where,

$S_{i j}^{1}=-\left(k_{k i j}^{*}-\omega^{2} m_{k i j}^{*}\right), \quad(i, j=1,2, \ldots, n)$.

As mentioned previously, $\mathbf{k}_{k}^{*}=\left(k_{k i j}^{*}\right)$ and $\mathbf{m}_{k}^{*}=\left(m_{k i j}^{*}\right)$ are independent of the exciting frequency $\omega$, therefore, just one calculation of $\mathbf{k}_{k}^{*}$ and $\mathbf{m}_{k}^{*}$ is required for the sensitivity analysis within the frequency domain $\left[\omega_{a}, \omega_{b}\right]$. Thus, the calculations will be much cheapter than the use of DFR method, when the sensitivities need to be calculated at many frequency points.

It should be noted that the mode-superposition technique used here is just a standard modedisplacement method. As discussed by e.g., Ma and Hagiwara (1991b), the mode-displacement method may lose accuracy when the mode number used in Eq. (74) is small, especially when the modes whose frequencies are lower than the exciting frequency need to be ignored (as it is sometimes necessary to solve the problem with respect to a higher exciting frequency). In this case, an improved mode-superposition technique presented by Ma and Higiwara (1991b) needs to be introduced to improve accuracy. 
It is noted that the sensitivity analysis methods mentioned in this section and last section can be extended for the case where the damping term is also considered. Some similar techniques as that presented in the Refs. (Ma and Hagiwara 1991a, b; 1992) can be used for this purpose.

\section{Examples}

In order to show the optimum design for the frequency response optimization problem described in above, we shall demonstrate three examples in plane stress elasticity. The first example is to find the optimal topology and shape from a given design domain, the second is for the optimal reinforcement design of an existing structure while the third is to minimize the frequency response within a given frequency domain. The objective function $f_{1}$ and the DFR sensitivity analysis method were used in the first two examples while the objective function and the MFR sensitivity analysis method were used in the third example.

Example 1. Optimal topology and shape problem. In the optimal topology and shape problem, only a design domain, boundary condition and exciting loads are given. As shown in Fig. 3, the design domain is specified as a rectangle, $8.0 \mathrm{~cm}$ in horizontal length and $5.0 \mathrm{~cm}$ in vertical height with two fixed support boundaries at A and B of the left end. A shear-like, period exciting load with the frequency $60 \mathrm{~Hz}$ is assumed to act on the center part $\mathrm{C}$ of the right end surface. This problem may be described as a shape optimization problem of a short cantilever beam subject to the transverse vibrating load at the free end. First, a finite-element model of the design domain is made by $640(20 \times 32)$ finite-elements with 693 nodes. Thus, we have 1386 D.O.Fs. for the structural domain and 1920 design variables for the optimization process in this problem.

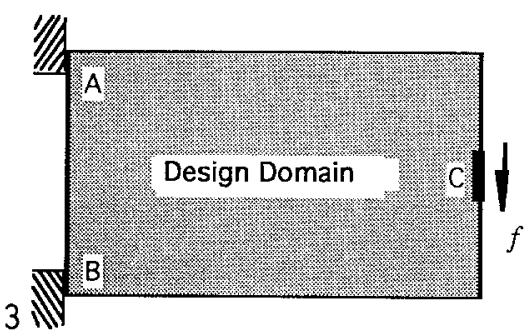

Fig. 3. Design domain of the optimization problem

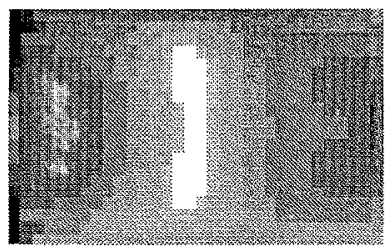

a

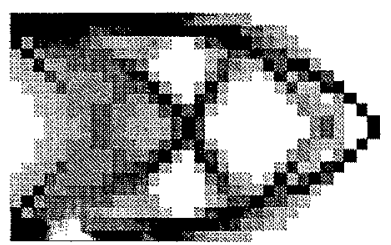

e

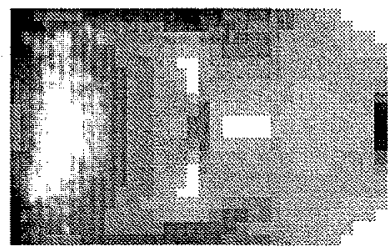

b

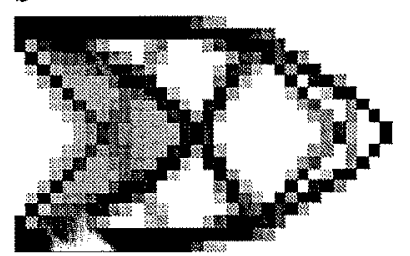

f

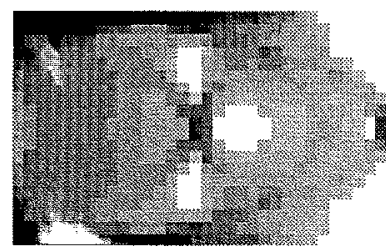

c

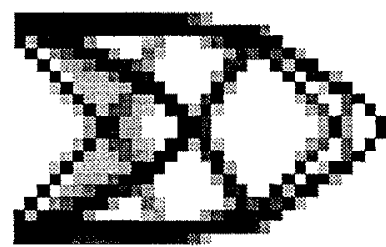

g

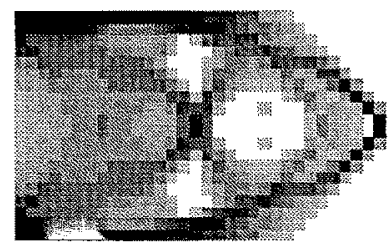

d

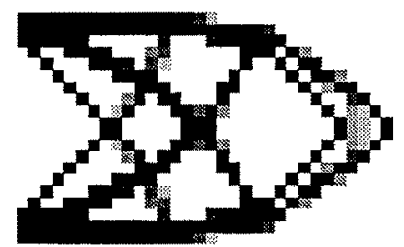

$\mathbf{h}$

Fig. $\mathbf{4 a}-\mathbf{h}$. Generation history of the optimal structure. ( $k$ : iteration number). $\mathbf{a} k=1, \mathbf{b} k=3, \mathbf{c} k=7, \mathbf{d} k=12$, e $k=18, \mathbf{f} k=25$, g $k=33$, h $k=150$ 
The optimization process is started from a given initial value of the design variable, i.e., $x_{i}^{0}=0.20(i=1,2, \ldots, N)$. The initial density of each element is $36 \%$, i.e., the initial structure fills $36 \%(14.4 / 40.0)$ of the volume of whole design domain. A volume constraint keeps this material volume at $36 \%$. Therefore, the optimization process becomes a matter of just moving material from one part of the design domain to the another part, without addition or reduction of the material.

Figure 4 gives the history of the optimal structure generation, which is calculated by the method presented in this paper. In this example, the initial value of the object function is 161.9 and final one become 113.8. As shown in Fig. 4, the material is dramatically moved during the optimization process and finally converges to an optimal structure.

To investigate the changes in the natural frequencies of the structure, a frequency analysis is performed in each iteration step. Figure 5 shows the variation history of the first and second natural frequencies. The frequencies of the initial structure were $25.8 \mathrm{~Hz}$ and $75.7 \mathrm{~Hz}$ and the final ones become $100.1 \mathrm{~Hz}$ and $100.2 \mathrm{~Hz}$. It is shown that when the structure is close to the optimal one, the first two natural frequencies are going to come together, until finally the optimal structure has repeated frequencies. This phenomenon is also found in other optimal design problems (e.g., Choi and Haug 1981).

Figure 6 shows the convergence history of the constraint function. As shown in Fig. 6, even though the initial value is started from zero, the constraint function has a minus value $-0.36(2.5 \%$
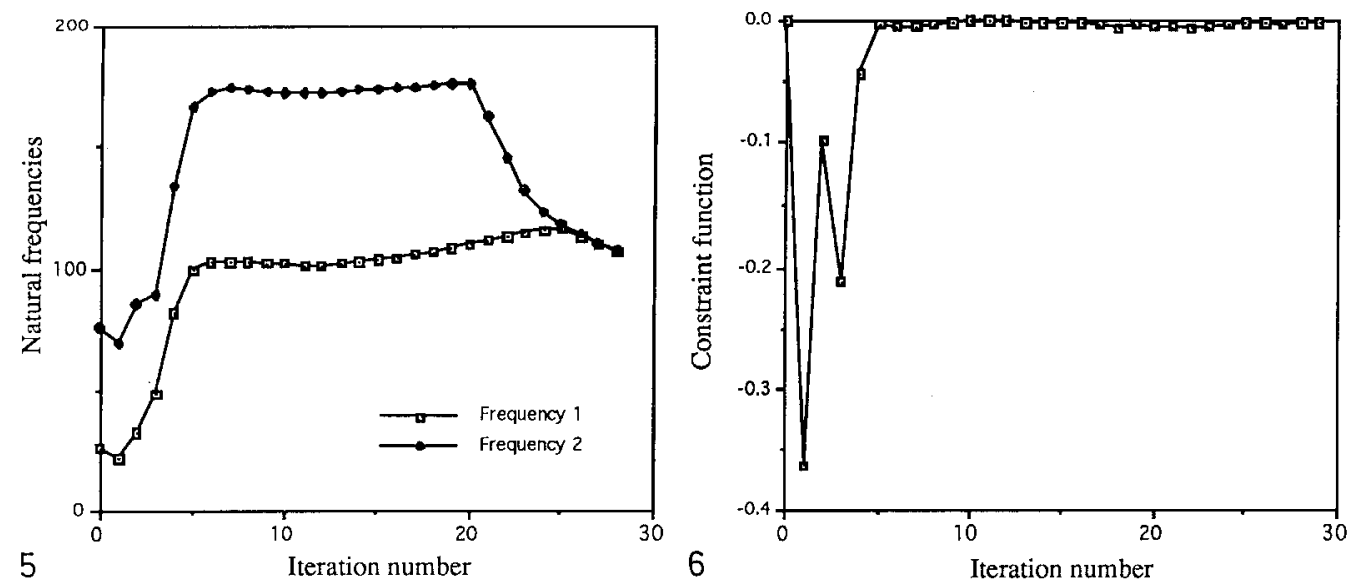

Figs. 5 and 6. 5 Variations in the first and second natural frequencies. 6 Convergence history of the constraint function
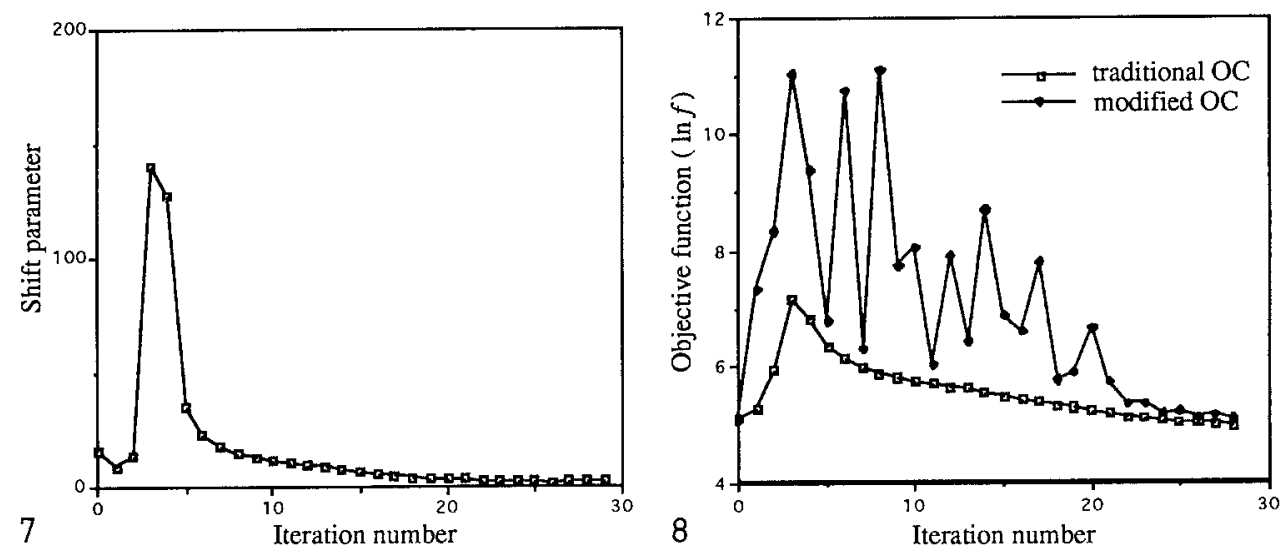

Figs. 7 and 8. 7 Variation of the shift parameter. 8 Comparison of the modified OC and the traditional OC (Dynamic case, $\omega=60.0 \mathrm{~Hz})$ 
of the given volume) at the first step. This is caused by the larger changes in the design variables, so that the approximate constraint function has a larger error from the primary one. But, after $k=30$, the constraint function becomes greater than $-0.002(0.14 \%$ of the given volume), and converges to zero as the iteration number $k$ is increased.

Figure 7 shows the variation history of the shift parameter $\mu$. It is shown that $\mu$ is almost monotonically reduced to zero as the iteration number is increased except at $k<5$. It should be noted that just one or two iterations are required in each step to calculate the Lagrange multiplier $\lambda^{*}$ using Eq. (64). This is also true for the all of the other trial examples provided that the iteration process is started from a properly (not specially) chosen initial value. Therefore, the calculation method of the Lagrange multiplier proposed in this paper is simple and also efficient.

A comparison of the results obtained either with or without shift parameter $\mu$ is given in Fig. 8. Figure 8 shows the convergence history of the objective function with respect to the first 30 iterations. There, the vertical coordinate is $\ln f_{1}$. As mentioned previously, if $\mu=0$, the method presented in this paper is reduced to the previous OC method. Figure 8 shows that a large oscillation occurs in the objective function when the previous OC method is used. Figure 9 shows that the optimization process did not converge to a physically sound structure. As shown in Fig. 8, if the modified OC method is used, the convergence of the objective function is smooth and
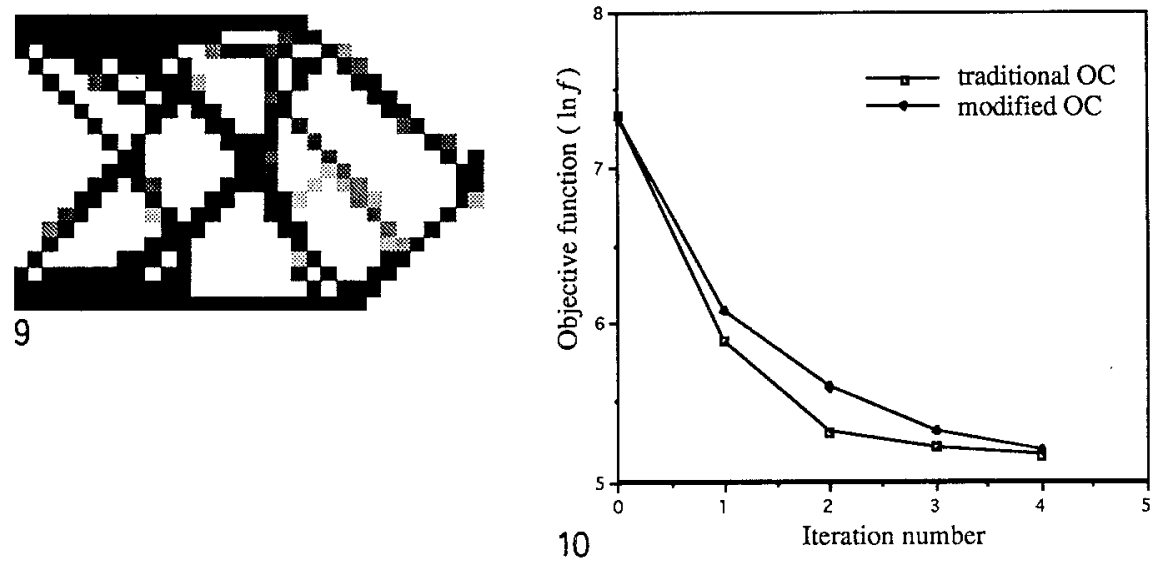

Figs. 9 and 10. 9 Final structure using the traditional OC. 10 Comparison of the modified OC and the traditional OC (Static case, $\omega=0.0 \mathrm{~Hz}$ )
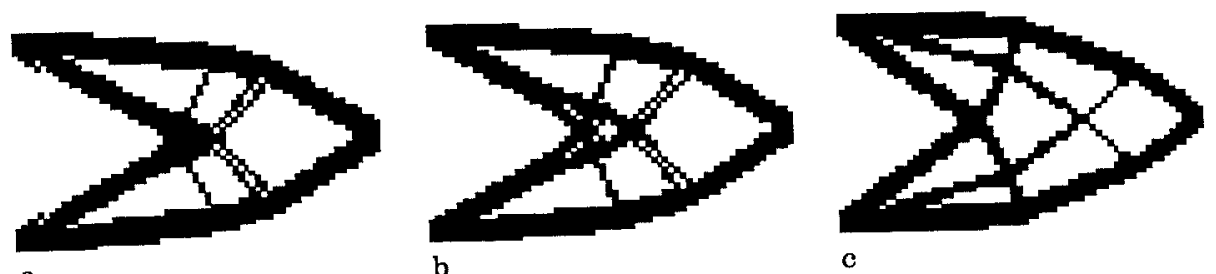

a

$\mathrm{b}$

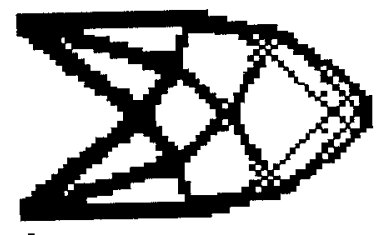

d

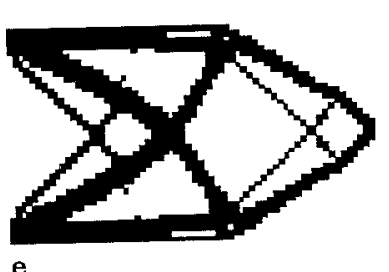

e

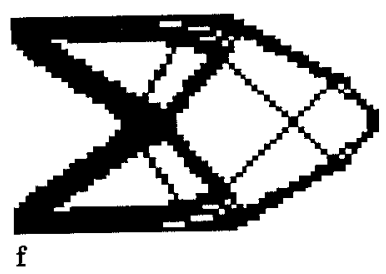

Fig. 11 a-f. Optimal topologies and shapes with respect to different exciting frequencies; $\mathbf{a} \omega=0 \mathrm{~Hz}\left(f_{1}=99.0\right) ; \mathbf{b} \omega=15 \mathrm{~Hz}$ $\left(f_{1}=100.6\right) ; \mathbf{c} \omega=30 \mathrm{~Hz}\left(f_{1}=104.5\right) ; \mathbf{d} \omega=45 \mathrm{~Hz}\left(f_{1}=113.6\right) ; \mathbf{e} \omega=60 \mathrm{~Hz}\left(f_{1}=122.7\right) ; \mathbf{f} \omega=75 \mathrm{~Hz}\left(f_{1}=137.6\right)$ 
monotonically decreasing except for the first four iterations. A big vibration of the objective function occurs in the first four iteration steps. This is caused by the resonant response while the first natural frequency of the structure crosses the exciting frequency $60 \mathrm{~Hz}$.

Figure 10 shows that even for the static problem, using the shift parameter (in this case $\mu$ has a minus value) could accelerate convergence. Some results show that a large positive $\mu$ values may slow down the convergence even though it can make the optimization process stable. Therefore, an investigation about how to actively control the shift parameter $\mu$ may required.

For obtaining more detailed topology and shape of the optimal structure, the design domain is decomposed by a refined mesh, $40 \times 64$ finite-elements. Each finite-element used in the previously analysis is divided into four elements. The initial value of the design variables is also changed to $x_{i}^{0}=0.18(i=1,2, \ldots, N)$ for the purpose of examining the influence of the initial condition. But, the other conditions are kept to be the same. Figure 11 shows the results of the optimum topology and shape optimization for the exciting frequencies $0 \mathrm{~Hz}, 15 \mathrm{~Hz}, 30 \mathrm{~Hz}, 45 \mathrm{~Hz}, 60 \mathrm{~Hz}$ and $75 \mathrm{~Hz}$, respectively. As shown in Fig. 11, in all of these cases, the optimization method proposed here converges well. The results show that the optimal topologies and shapes are very different when the exciting frequency is changed while refinement of finite element model does not alter the optimum layout significantly. It implies that the optimal topology and shape obtained in the static optimization is not optimal in the dynamic case. Comparison of Fig. 4(h) and Fig. 11(e) shows that the result obtained in this example is not much mesh dependent nor initial condition dependent. But, this feature may not be always true, especially when the structure is not statically determinate or the initial condition has a larger change. This kind of problems should be discussed in future.

Example 2. Optimal reinforcement problem. In the optimal reinforcement problem, a core of the structure is specified at beginning as shown in Fig. 12. Here the core-structure is not changed in the optimization process. The material is only added to reinforce the core-structure within the design domain.

Figure 13 shows the result of the optimum reinforcement with respect to the exciting frequencies $20 \mathrm{~Hz}, 50 \mathrm{~Hz}, 60 \mathrm{~Hz}$ and $70 \mathrm{~Hz}$. As shown there, the optimization method does converge,

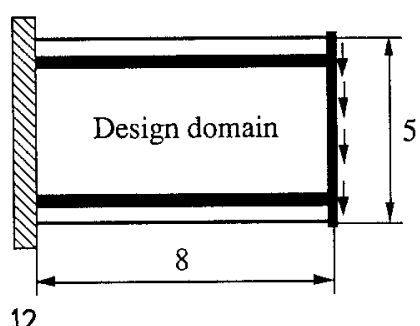

12

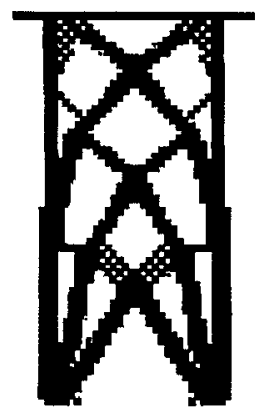

a

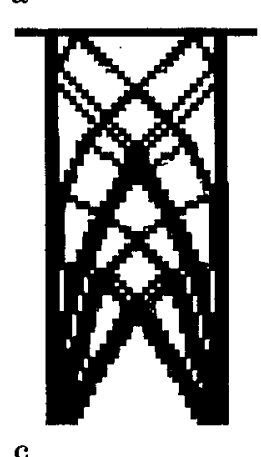

13

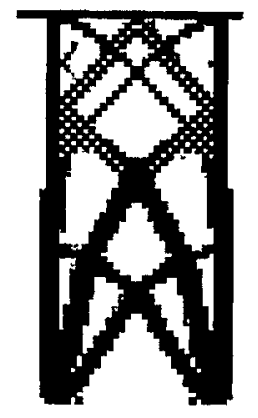

b

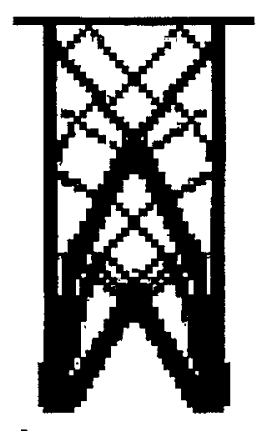

d

Figs. 12 and 13. 12 Design domain of the optimal reinforcement problem. 13a-d Results of optimal reinforcements; $\mathbf{a} \omega=20 \mathrm{~Hz}, \mathbf{b} \omega=$ $50 \mathrm{~Hz}, \mathrm{c} \omega=60 \mathrm{~Hz}, \mathrm{~d} \omega=70 \mathrm{~Hz}$ 


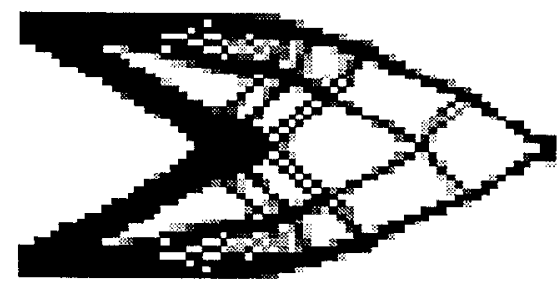

14

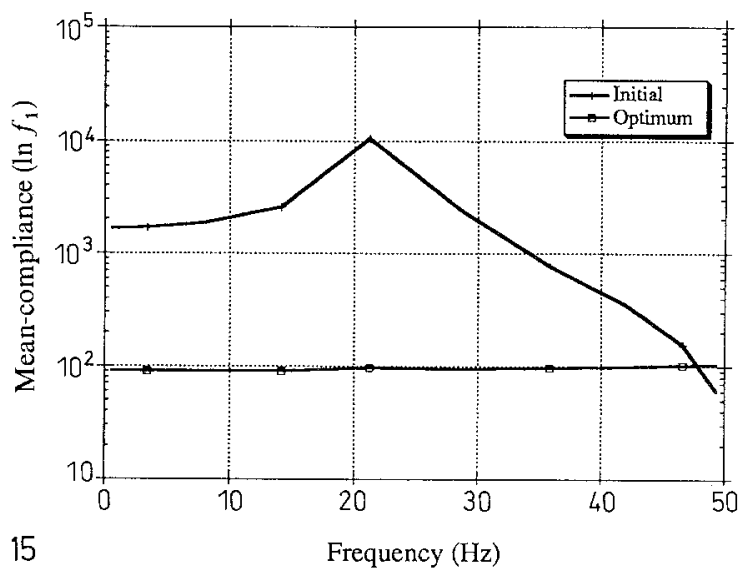

Figs. 14 and 15. 14 Optimal structure subjected to the given frequency domain. 15 Reduction of the objective function

and the optimal reinforcement layouts are very different when the exciting frequency is changed. This implies that we have to make different reinforcements for the problems with different exciting frequencies.

Example 3. Frequency domain problem. The last example is to show the result about minimizing the integral of the mean-compliance over a given frequency domain. The design problem is same as the problem which is discussed in Example 1 except we consider an integration here. Here, the fine mesh, $(40 \times 64)$ finite element model, is used in this problem. The integral domain is considered $0 \mathrm{~Hz}$ to $50 \mathrm{~Hz}$, and ten Gauss points are used for the Gaussian Quadrature Eq. (19). For the frequency response analysis and sensitivity analysis, the modal superposition technique, Eq. (74), and MFR sensitivity analysis method, Eq. (80), are utilized, where the first eight modes are employed for this example. Also, for the optimization process, the start points are $x_{i}^{0}=0.18$ $(i=1,2, \ldots, N)$.

Figure 14 gives the optimal structure obtained using the method presented in this paper. Figure 15 shows the mean-compliance of the initial design domain and the optimal structure within the frequency domain $(0,50 \mathrm{~Hz})$. It is seen that a great reduction of the mean-compliance can be obtained by using the method presented in this paper.

\section{Conclusion}

A topology and shape optimization method using the conception of OMD is developed for the frequency response problem of a vibrating structure. It is shown that the use of this method makes it possible to obtain the optimal structure, which is not only optimal in size and shape but also in topology. To solve the optimization problem defined in this paper, a modified OC algorithm is developed using the convex approximation approach and the dual method. It is shown that this algorithm is very efficient and well convergent for the problem.

\section{References}

Bendsøe, M. P. (1989): Optimal shape design as a material distribution problem. Struct. Optimization 1, $193-202$

Bendsøe, M. P.; Kikuchi, N. (1988): Generating optimal topologies in structural design using a homogenization method. Comput. Methods Appl. Mech. Energ. 71, 197-224

Bendsøe, M. P.; Diaz, A.; Kikuchi, N. (1992): Topology and generalized layout optimization of elastic structures. In: Bendsøe, M. P.; Soates, C. A. M. (eds): Topology design of structures, pp. 159-205. NATO ASI Series, Kluwer

Berke, L.; Venkayya, V. B. (1974): Review of optimality criteria approaches to structural optimization. In: Schmit, L. A. (ed.): Structural optimization symposium, pp. 23-34. New York 
Choi, K. K.; Haug, E. J. (1981): Optimization of structures with repeated eigenvalues. In: Haug, E. J.; Cea, J. (eds.): Optimization of distributed parameter structures Volume I, pp. 219-277. The Netherlands: Sijthoff \& Noordhoff

Diaz, A.; Kikuchi, N. (1992): Solutions to shape and topology eigenvalue optimization problems using a homogenization method. Preprint, Dept. of Mech. Engng., Michigan State University, East Lansing, MI

Fleury, C.; Braibant, V. (1986): Structural optimization: a new dual method using mixed variables. Internat. J. Numer. Methods Engrg. 23, 409-428

Haftka, R. T.; Grandhi, R. V. (1986): Structural shape optimization-a survey. Comput. Methods. Appl. Mech. Energ. 57, 91-106

Haftka, R. T.; Gurdal, Z. (1992): Elements of structural optimization. Third revised and expanded edition. The Netherlands: Kluwer

Kirsch, U. (1989): Optimum topologies of structures. Appl. Mech. Rev. 42, 223-239

Ma, Z.-D.; Hagiwara, I. (1991a): Sensitivity analysis methods for coupled acoustic-structural systems, part 2: direct frequency response and its sensitivities. AIAA Journal 29, 1796-1801

Ma, Z.-D.; Hagiwara, I. (1991b): Improved mode-superposition technique for modal frequency response analysis of coupled acoustic-structural systems. AIAA Journal 29, 1796-1801

Ma, Z.-D.; Hagiwara, I. (1992): Sensitivity calculation methods for modal frequency response of coupled acoustic-structural system. JSME International Journal Series III, 35, 12-21

Olhoff, N.; Bendsøe, M. P.; Rasmussen, J. (1991): On CAD-integrated structural topology and design optimization. Comput. Methods Appl. Mech. Energ. 89, 259-279

Olhoff, N. N.; Taylor, J. E. (1983): On structural optimization. J. Appl. Mech. 50, 1134-1151

Rozvany, G. I. N. (1992): Layout theory for grid-type structures. In: Bendsøe, M. P.; Soates, C. A. M. (eds): Topology design of structures, pp. 251-272. NATO ASI Series, The Netherlands: Kluwer

Suzuki, K.; Kikuchi, N. (1990): Generalized layout optimization of shape and topology in three-dimensional shell structures. Rept. No. 90-05, Dept. Mich. Engrg. and Appl. Mech., Comp. Mech. Lab., University of Michigan, USA

Suzuki, K.; Kikuchi, N. (1991): A homogenization method for shape and topology optimization. Comput. Methods Appl. Mech. Energ. 93, 291-318

Sanchez-Plencia, E. (1980): Non-homogeneous media and vibration theory. Lecture notes in physica 127, Berlin, Heidelberg, New York: Springer

Svanberg, K. (1987): The method of moving asymptotes-a new method for structural optimization. Internat. J. Numer. Methods Engrg. 24, 359-373

Communicated by S. N. Atluri, May 28, 1993 